\title{
SELF-EMBEDDING VIDEO WATERMARKING USING DUAL ORTHOGONAL COMPLEX CONTOURLET TRANSFORM WITH AUTOCORRELATION SYSTEM
}

U. R. Padma ${ }^{* 1}$, J. Jayachitra ${ }^{2}$

${ }^{* 1}$ M.E Computer Science and Engineering, IFET college of Engineering, INDIA

${ }^{2}$ Associate professor, IFET college of Engineering, INDIA

Abstract:

This paper presents a novel non-blind watermarking algorithm using dual orthogonal complex contourlet transform. The dual orthogonal complex contourlet transform is preferred for watermarking because of its ability to capture the directional edges and contours superior to other transforms such as cosine transform, wavelet transform, etc. Digital image and video in their raw form require an enormous amount of storage capacity and the huge data systems also contain a lot of redundant information. Compression also increases the capacity of the communication channel. Image Compression using SPIHT Set Partitioning in Hierarchical Trees algorithm based on Huffman coding technique. SPIHT algorithm is the lossless compression algorithms reduce file size with no loss in image quality and comparing the final results in terms of bit error rate, PSNR and MSE.

Keywords:

Compressed video, data embedding, tampering, contourlet transform, SPIHT.

Cite This Article: U. R. Padma, and J. Jayachitra, "Self-Embedding Video Watermarking Using Dual Orthogonal Complex Contourlet Transform With Autocorrelation System" International Journal of Research - Granthaalayah, Vol. 3, No. 4(2015): 89-98.

\section{INTRODUCTION}

Digital watermarking is the process of embedding information into a digital signal in a way that is difficult to remove. The process of digital watermarking involves the modification of the original multimedia data to embed a watermark containing key information such as authentication or copyright codes. Watermarking represents an efficient technology for ensuring data integrity and data-origin authenticity.

Watermarking is the process of embedding data into multimedia element can primarily for copyright protection. Because of its Growing popularity, the Discrete Wavelet Transform (DWT) is commonly used in the watermarking scheme increase, power consumption [1]. Digital video watermarking is the process to embedding a digital code into digital video sequences. Digital video is nothing but a sequence of consecutive still images.

Digital video watermark some most important properties of watermarking process are required. Such as, Robustness, Imperceptibility, Unambiguous, Loyalty, Computational Cost, 


\section{INTERNATIONAL JOURNAL of RESEARCH -GRANTHAALAYAH

Interoperability, CBR (Constant Bit Rate), Random detection and Blind detection scheme [2]. The watermark should be able to survive any attacks like compression, scaling, noise, filtering, etc. The video watermarking is adopted either to compressed or to uncompressed (sometimes it's also called raw) domain. A video can either watermarked either in the RAW format or in compressed format.

The digital video watermarking by treating each video frames as images. All the properties and effects of image watermarking are directly extensible to the video watermarking. The distribution of the watermark in multiple frames is order to maintain the statistical and perceptual invisibility. A digital watermarking method to be effective it should be imperceptible, and robust to common image manipulations like compression, filtering, rotation, scaling cropping, and collusion attacks among many other digital signal processing operations. Current digital image watermarking techniques can be grouped into two major classes: spatial-domain and frequencydomain watermarking techniques. Compared to spatial domain technique, frequency-domain watermarking techniques proved to be more effective with respect to achieving the imperceptibility and robustness requirements of digital watermarking algorithms [3].

\section{LITERATURE SURVEY}

Sonal Jain, Brajlata Chourasiya [4] proposes a new data-hiding method in the motion vectors of MPEG compressed video. Based on the selection of attributes of motion vectors whose associated macroblocks prediction error is high and low PSNR for hiding a bit in each of their horizontal and vertical components. L. Sumalatha, G. RoslineNesa Kumari, V.Vijaya Kumar [5] Proposes a simple block based content checksum watermarking (BCCW) method for image authentication and tamper localization. BCCW is a hierarchical and block based method the image is divided into sub blocks of size $4 \times 4$. A hierarchical relationship is established by dividing each $4 \times 4$ as a set of four $2 \times 2$ blocks.

Sajjad Dadkhah, Azizah Abd Manaf and Somayeh Sadeghi [6] propose an efficient image tamper detection method using 3 LSB (last significant bit) watermarking technique which is able to authenticate the digital image and detect the tamper locations accurately. Ammar M. Hassan, Yassin M. Y. Hasan, Mohamed A. A. Wahab [7] proposes a new vector quantization (VQ) attack that is applicable on self-recovery image authentication. The counterfeiting attack, the codebook is developed to contain authenticated blocks with their encrypted codes and displacement vector(s). To fabricate a counterfeit image that can pass the verification process as an authentic image, the attacker can search the codebook and find the best match for each unauthenticated block of an arbitrary image.

P. MeenakshiDevi, M. Venkatesan and K. Duraiswamy [8] Proposes a wavelet-based fragile watermarking scheme for secure image authentication. The image is decomposed by integer Haar wavelet transform. The watermark is embedded only in the $\mathrm{HH}$ sub-band. The embedded watermark is generated and scrambled based on the size of the image to be watermarked. This provides more protection to the watermarking system. Integer wavelet transform is applied and 


\section{INTERNATIONAL JOURNAL of RESEARCH -GRANTHAALAYAH

localizes the tampering at pixel level. The security of the whole system against VQ attack is achieved by permutation of wavelet coefficients.

In existing system Moment-preserving technique is able to classify the pixels of a given image into two groups. The pixels in each group are assigned to a certain gray value such that the moment of the image can be preserved. In this technique, a threshold is selected to group the pixels with the gray values above the threshold, and other pixels with gray values equal to and below the threshold. Bi-level moment-preserving technique is the simplest one, where only one threshold is determined to classify the pixels with the grey values above the threshold, and other pixels with grey values equal to or below the threshold. The pixels of the two groups are then assigned to two representative grey values, respectively, to obtain the thresholded image preserving the same moment. The bi-level method used to preserve the moment of the image is incorporated into the proposed self-embedding watermarking scheme such that the tampered region of the color image can be recovered with high quality while the proofing result is obtained [9].

\section{DISADVANTAGES OF EXISTING ALGORITHM}

1. Every pixel affects every other pixel, but if the DCT is used instead of the DFT, values of the pixels come directly from the transform of the time domain value.

2. In DCT watermarking, after embedding the cover object contains many noises like random noise, Gaussian noise and salt and pepper noise.

3. Only spatial correlation of the pixels inside the single 2-D block is considered and the correlation from the pixels of the neighboring blocks is neglected.

4. Impossible to completely de-correlate the blocks at their boundaries using DCT.

5. Undesirable blocking artifacts affect the reconstructed images or video frames.

\section{PROPOSED WORK}

In the proposed system Dual Orthogonal complex contourlet Transform is used to identify the tamper location in the self-embedded video watermarking with autocorrelation. The Dual orthogonal complex Contourlet Transform performs decomposition in two stages namely the Laplacian pyramid (LP), Directional Filter Bank (DFB) respectively. In the first stage of decomposition LP is used to capture point discontinuities, followed by DFB in the second stage to link point discontinuities into linear structure. Laplacian pyramid is a method for achieving multiscale decomposition.

The function of LP at each step is to decompose the image to generate a sampled low pass version of the original image where the difference between the original image and the prediction giving a bandpass image. Source information is partitioned into Multiple Descriptions Coding (MDC) as odd and even pixel intensities (two descriptions) of host image. The receiver is able to reconstruct the original source within some prescribed distortion levels by using few of the descriptions based on odd and even pixel intensities of host image. SPIHT is computationally 


\section{INTERNATIONAL JOURNAL of RESEARCH -GRANTHAALAYAH

very fast and among the best image compression algorithms known today. According to statistic analysis a simple and effective method combined with Huffman encode for further compression. SPIHT codes the individual bits of the image wavelet transform coefficients following a bitplane sequence.

\section{ARCHITECTURE FOR PROPOSED SYSTEM}

The block diagram describes the flow of the proposed system. Initially video is taken as input. Video contain sequence of frames. Frame conversion using filter based i.e. weighted median filter. FRC (Frame Rate Conversion) are used in compression, video format conversion, quality enhancement, stereo vision, etc. The 24 FPS rate became the de facto standard for sound motion pictures and videos. The proposed system comprises of the following modules

1. Filtering using Adaptive median filter.

2. Conversion of Spatial domain image to frequency domain image.

3. Image embedding using Auto-correlation.

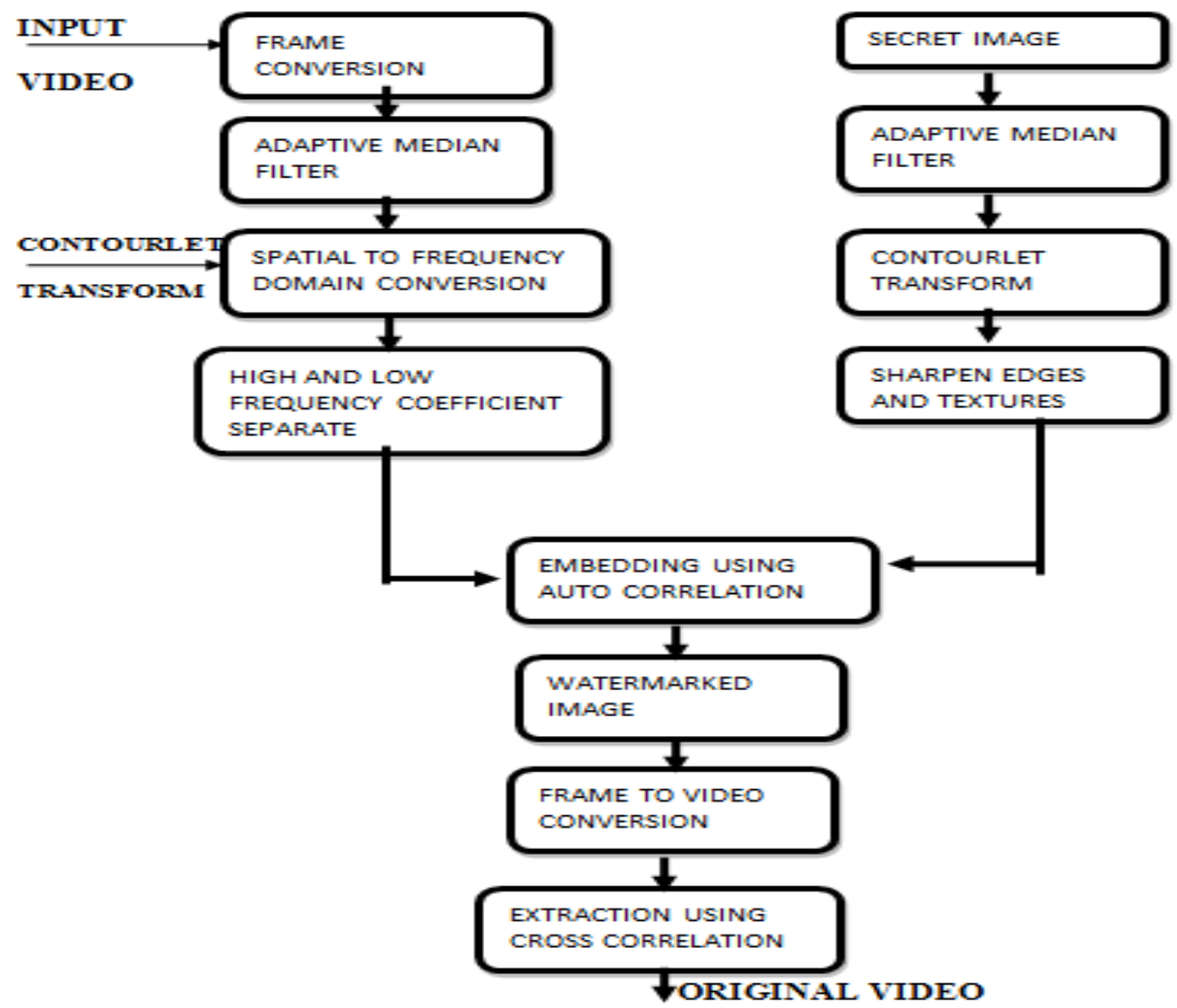

Fig 1: work flow for proposed system 


\section{INTERNATIONAL JOURNAL of RESEARCH -GRANTHAALAYAH \\ A knowledge Repository}

Science

\section{SIMULATION RESULT}

The .Avi video is divided into frames and secret image are divided into block based on the algorithm. Each secret image block is hidden into each selected frames. The longest video is taken its length is 31 seconds. Video contain 1100 frames and secret image split into 32 frames. Embedded video with secret image is the final output.

SPIHT algorithm is the lossless compression algorithms reduce file size with no loss in image quality. When the file is saved it is compressed, when it is decompressed (opened) the original data is retrieved. This type of compression can be applied not just to graphics but to any kind of computer data such as spreadsheets, text documents and software applications.

\section{ORIGINAL VIDEO}

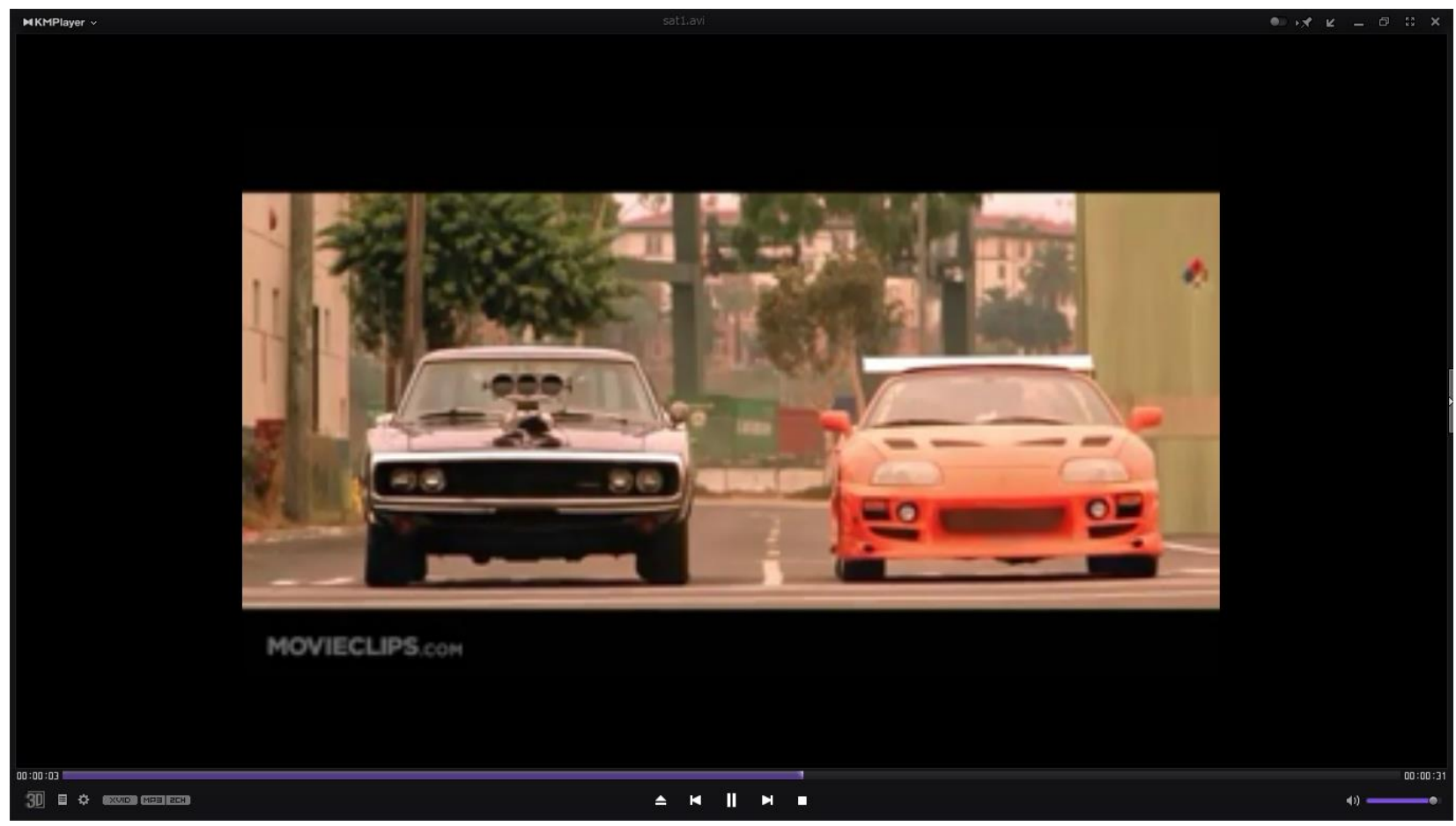


S

ग्रन्थलिया:

Science
INTERNATIONAL JOURNAL of RESEARCH -GRANTHAALAYAH

A knowledge Repository
口为䡮回

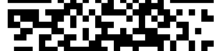

.

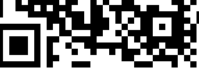

\section{VIDEO TO FRAMES}

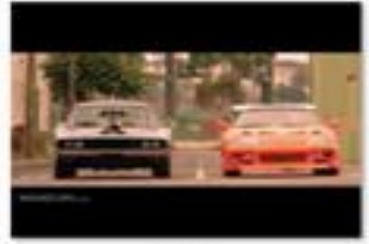

ming

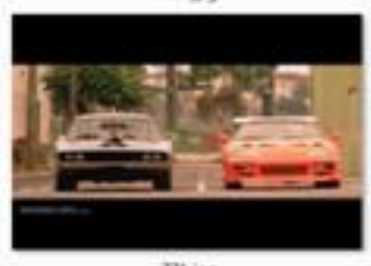

J2tiog

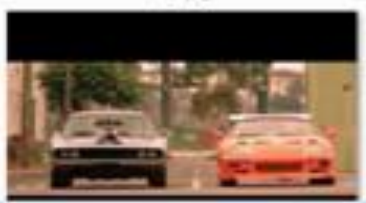

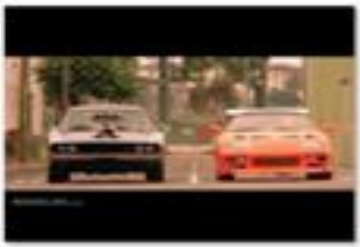

numps

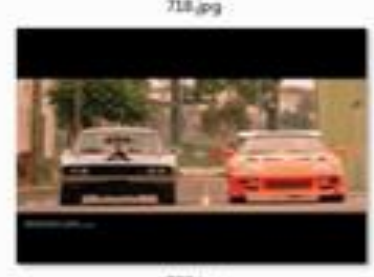

22200

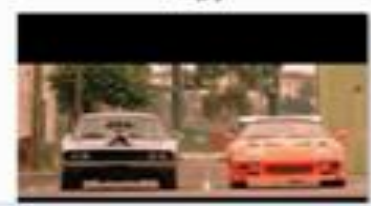

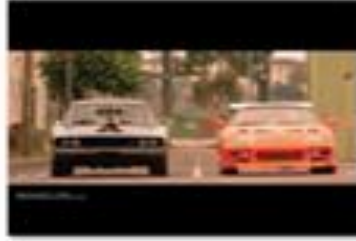

7899

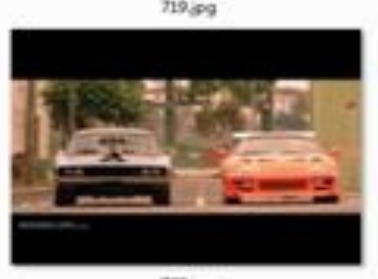

$223 \mathrm{pg}$

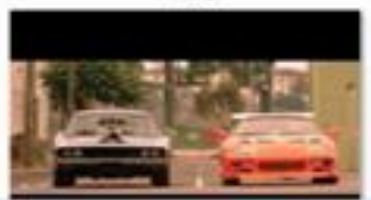

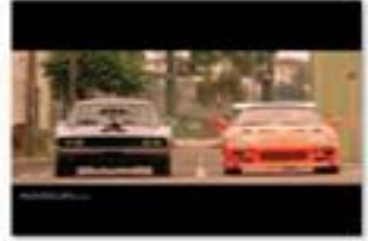

720 ing

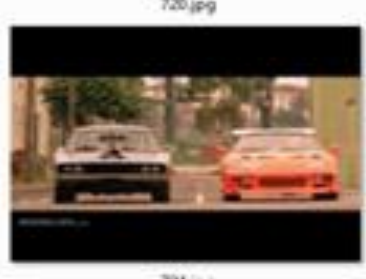

rains

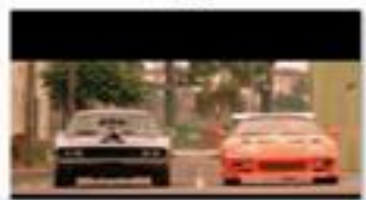

\section{SECRET IMAGE}

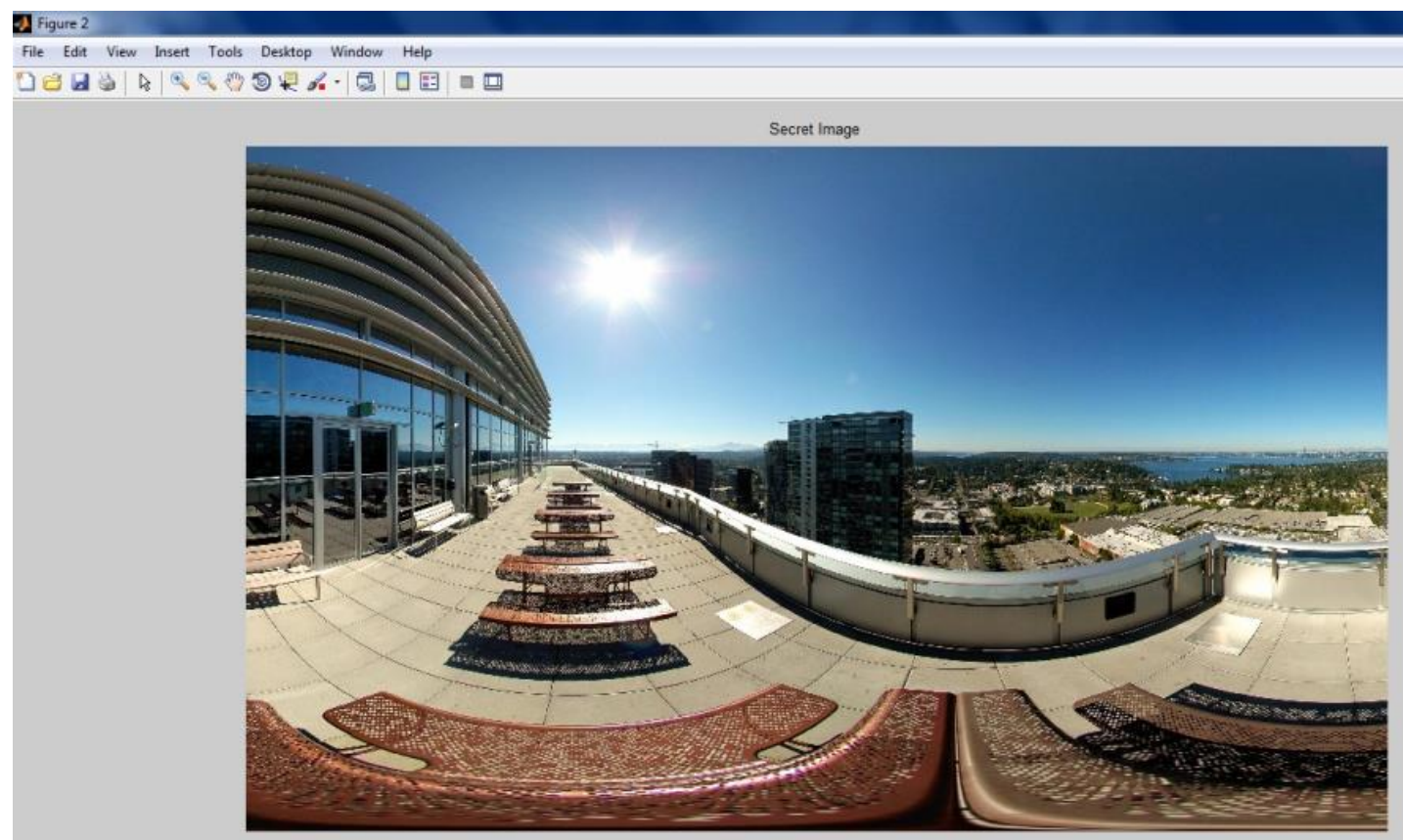




\section{SECRET IMAGE TO 32 BLOCKS}

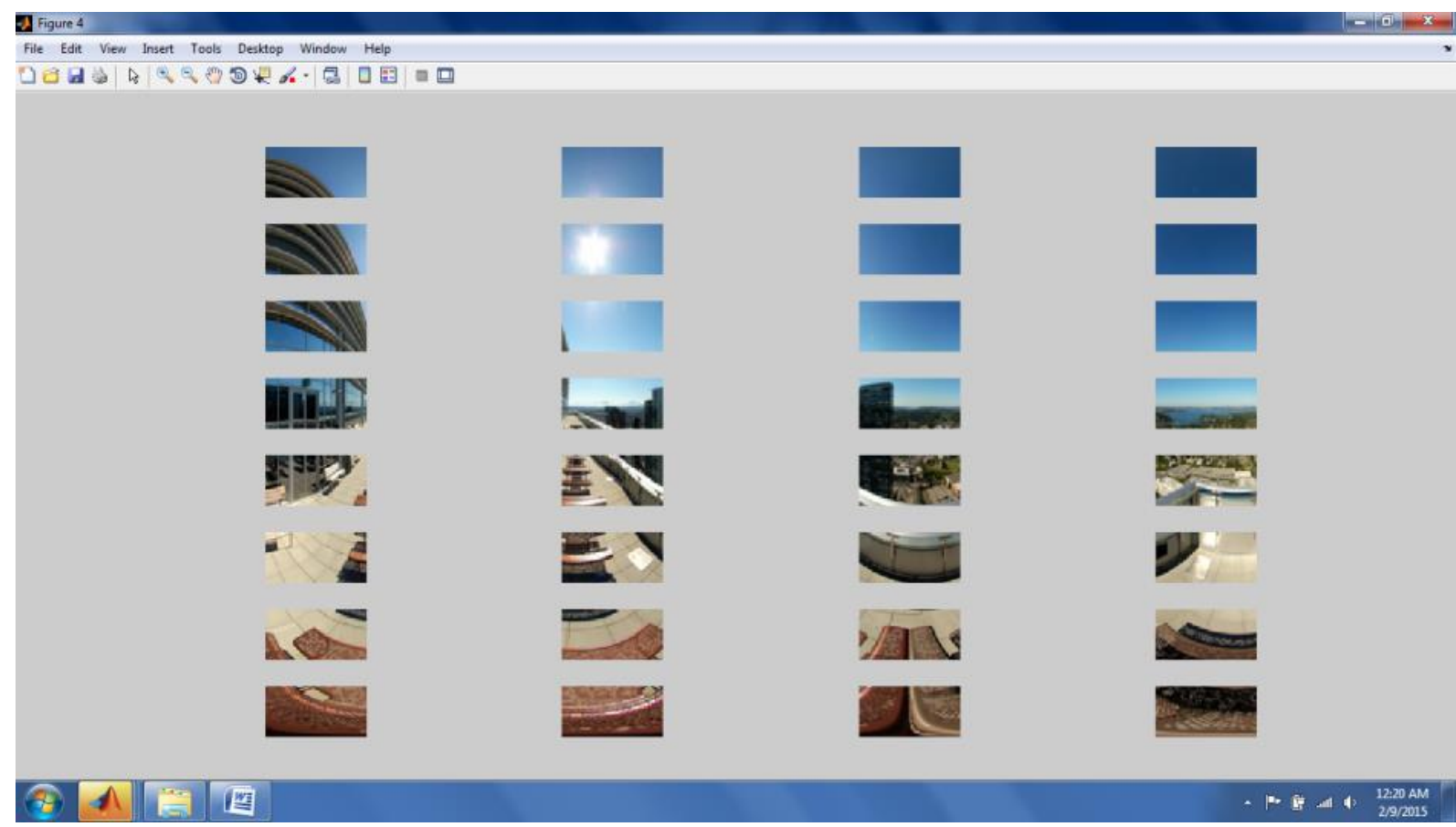

SPATIAL DOMAIN TO FREQUENCY DOMAIN CONVERSION

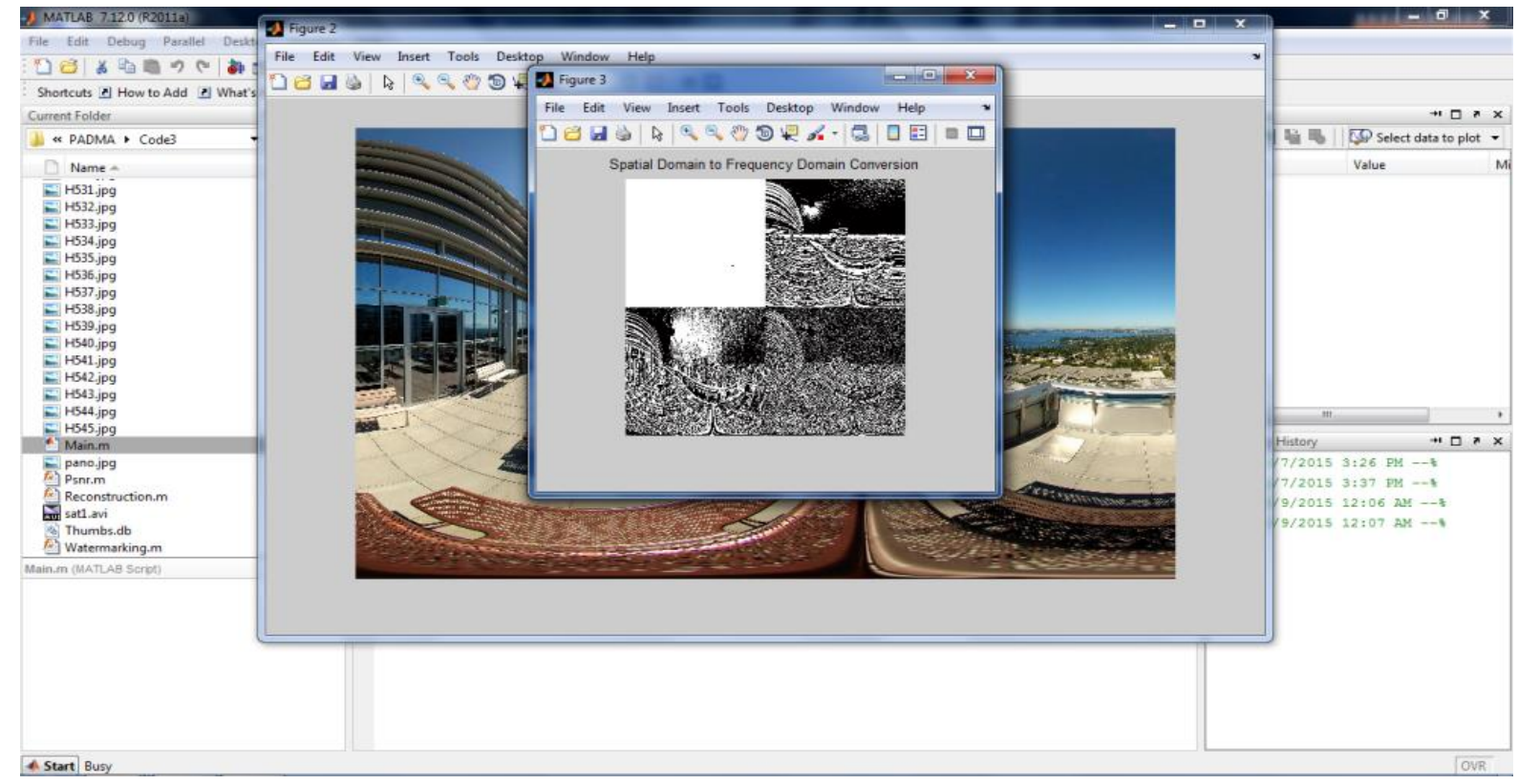




\section{INTERNATIONAL JOURNAL of RESEARCH -GRANTHAALAYAH}

ग्रवर्थालया

\section{EXTRACTED SECRET IMAGE}

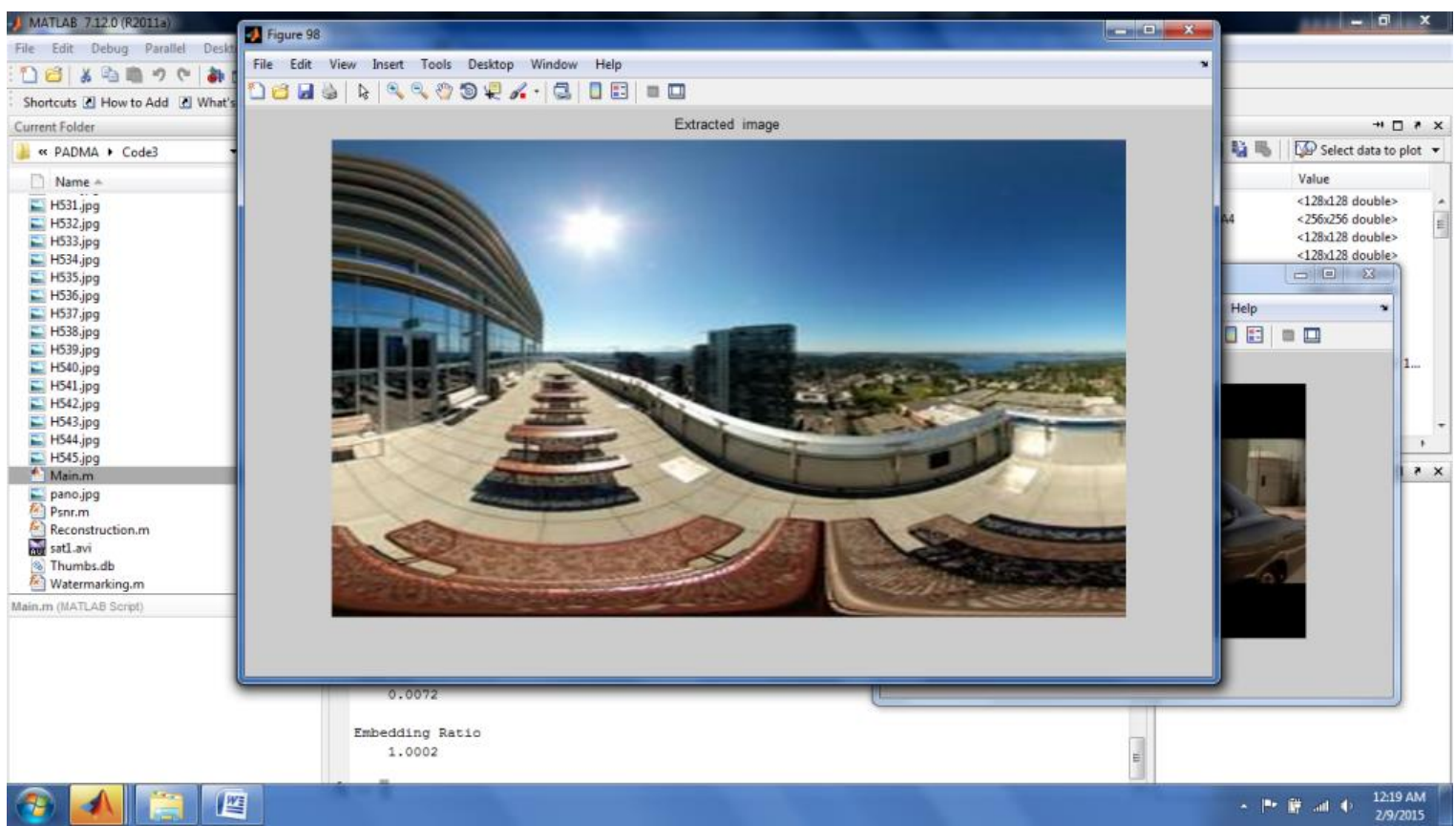

\section{FINAL RESULT}

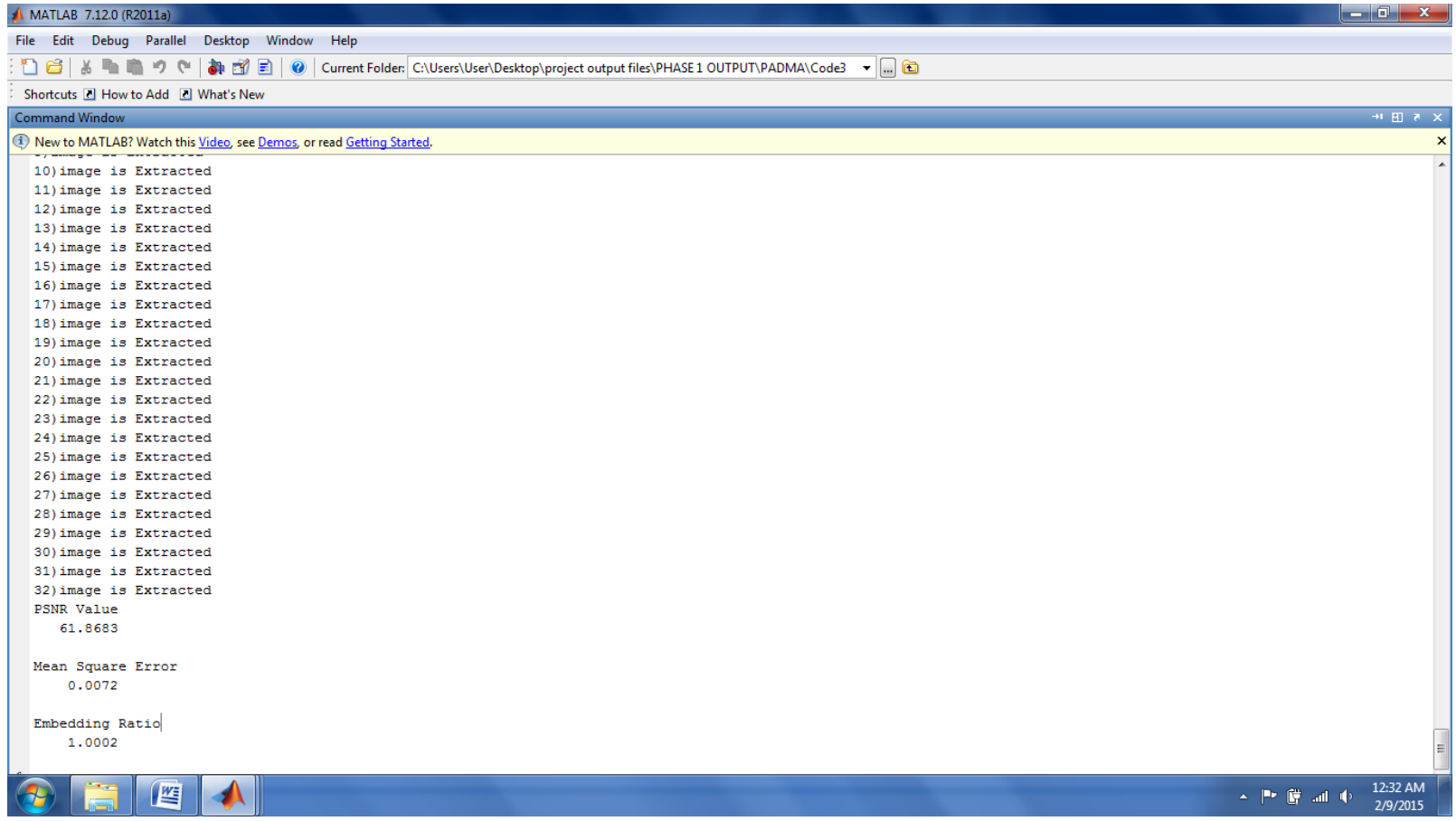




\section{INTERNATIONAL JOURNAL of RESEARCH -GRANTHAALAYAH \\ A knowledge Repository}

Science

\begin{tabular}{|l|l|l|l|l|l|l|}
\hline S.NO & $\begin{array}{l}\text { VIDEO } \\
\text { (.avi) }\end{array}$ & $\begin{array}{l}\text { SECRET } \\
\text { IMAGE } \\
\text { (jpg) }\end{array}$ & $\begin{array}{l}\text { PSNR } \\
\text { VALUE }\end{array}$ & MSE & $\begin{array}{l}\text { EMBEDDING } \\
\text { RATIO }\end{array}$ & $\begin{array}{l}\text { RESOLUTION } \\
\text { PIXEL }\end{array}$ \\
\hline 1. & sat1 & pano & 61.8683 & 0.0072 & 1.0002 & 16200000 \\
\hline 2. & car & blue & 62.0193 & 0.0071 & 1.0002 & 15300000 \\
\hline 3. & viptraffic & abc & 61.598 & 0.0072 & 1.0002 & 15552000 \\
\hline
\end{tabular}

Table 1: Various list of videos and secret images performance metrics

PSNR, MSE are two error metrics used to compare image compression quality.

The PSNR ratio is often used as a quality measurement between the original and a compressed image. Higher the PSNR better the quality of the compressed or reconstructed image. It represents a measure of peak error. Higher PSNR better degraded image has been reconstructed to match the original image and the better the reconstruction mechanism. PSNR over a converted luminance or gray scale channel as the eye is generally 4 times more susceptible to luminance changes as opposed to change in chrominance.

MSE is cumulative squared error between the compressed and the original image, lower value of MSE, the lower of the error. Minimize the MSE between images with respect the maximum signal value of the image. For color images MSE is taken over all pixels values of each individual channel and is averaged with the number of color channels.

\section{CONCLUSION}

The development of effective digital image copyright protection methods have recently become an urgent and necessary requirement in the multimedia industry due to the ever-increasing unauthorized manipulation and reproduction of original digital objects. Multimedia copyright protection and authentication is a major challenge in multimedia industry. To overcome these challenges proposed system has solution to solve these issues. Embedded video is compressed to reduce the size for storage purposes.

\section{REFERENCES}

[1] Anumol T.J, P Karthigaikumar "DWT based Invisible Image Watermarking Algorithm for Color Images" in the proceedings of IJCA Special Issue on "Computational Science - New Dimensions \& Perspectives" NCCSE, 2011. 


\section{INTERNATIONAL JOURNAL of RESEARCH -GRANTHAALAYAH \\ A knowledge Repository}

Science

[2] Archana Srivastava, "Enhancement of Robustness of Color Video Watermarking using 2D$D W T$ " in the proceedings of International Journal of Computer Science \& Engineering Technology (IJCSET).

[3] Nikita Kashyap, G. R. SINHA, "Image Watermarking Using 3-Level Discrete Wavelet Transform (DWT)" in the proceedings of I.J.Modern Education and Computer Science, 2012.

[4] Sonal Jain, Brajlata Chourasiya "A Secure Process of Hiding Data in Motion Vector of Compressed Video Based on Artifacts" in the proceedings of International Journal of Recent Technology and Engineering (IJRTE) ISSN: 2277-3878, Volume-2, Issue-5, November 2013.

[5] L. Sumalatha, G. RoslineNesa Kumari, V.Vijaya Kumar "A Simple Block Based Content Watermarking Scheme for Image Authentication and Tamper Detection" in the proceedings of International Journal of Soft Computing and Engineering (IJSCE) ISSN: 2231-2307, Volume-2, Issue-4, September 2012.

[6] Sajjad Dadkhah, Azizah Abd Manaf and Somayeh Sadeghi "Efficient Digital Image Authentication and Tamper Localization Technique Using 3Lsb Watermarking" in the proceedings of IJCSI International Journal of Computer Science Issues, Vol. 9, Issue 1, No 2, January 2012 ISSN (Online): 1694-0814.

[7] Ammar M. Hassan, Yassin M. Y. Hasan, Mohamed A. A. Wahab "A New Vector Quantization Attack on Self-Recovery Image Authentication” in the proceedings of ICCIT 2012.

[8] MeenakshiDevi, M. Venkatesan and K. Duraiswamy "A Fragile Watermarking Scheme for Image Authentication with Tamper Localization Using Integer Wavelet Transform" in the proceedings of Journal of Computer Science 5 (11): 831-837, 2009 ISSN 1549-3636.

[9] Kuo-Cheng Liu, Information Educating Center, Taiwan Hospitality \& Tourism College, Taiwan "Self-embedding watermarking scheme for colour images by bi-level moment-preserving technique" in the proceedings of Published in IET Image Processing Received on MAY 2012 , ISSN 1751-9659. 